\title{
Phyllosphere yeasts rapidly break down biodegradable plastics
}

\author{
Hiroko K Kitamoto ${ }^{1 *}$, Yukiko Shinozaki ${ }^{1}$, Xiao-hong Cao ${ }^{1}$, Tomotake Morita ${ }^{2}$, Masaaki Konishi ${ }^{2,5}$, Kanako Tago ${ }^{1}$, \\ Hideyuki Kajiwara ${ }^{3}$, Motoo Koitabashi', Shigenobu Yoshida', Takashi Watanabe', Yuka Sameshima-Yamashita', \\ Toshiaki Nakajima-Kambe ${ }^{4}$ and Seiya Tsushima'
}

\begin{abstract}
The use of biodegradable plastics can reduce the accumulation of environmentally persistent plastic wastes. The rate of degradation of biodegradable plastics depends on environmental conditions and is highly variable.

Techniques for achieving more consistent degradation are needed. However, only a few microorganisms involved in the degradation process have been isolated so far from the environment. Here, we show that Pseudozyma spp. yeasts, which are common in the phyllosphere and are easily isolated from plant surfaces, displayed strong degradation activity on films made from poly-butylene succinate or poly-butylene succinate-co-adipate. Strains of $P$. antarctica isolated from leaves and husks of paddy rice displayed strong degradation activity on these films at $30^{\circ}$ C. The type strain, P. antarctica JCM 10317, and Pseudozyma spp. strains from phyllosphere secreted a biodegradable plastic-degrading enzyme with a molecular mass of about $22 \mathrm{kDa}$. Reliable source of biodegradable plastic-degrading microorganisms are now in our hands.
\end{abstract}

Keywords: Pseudozyma, Biodegradable plastic, Phyllosphere, Yeast

\section{Introduction}

For the last 60 years, the use of synthetic polymers has grown progressively because of their low cost, reproducibility, and resistance to physical aging and biological attack. However, devices and materials made of synthetic polymers are sometimes used for short-term applications and are subsequently disposed at high rates to the natural environment; ideally such items should be biodegradable so that they do not accumulate in the environment. Biodegradable plastics are a family of polymer products with a molecular structure that is susceptible to biological decomposition into benign or even beneficial products. The environmentally beneficial perception of these materials, and their range of potential applications is expandin: it already extends to composting bags, mulch films, packaging of agricultural supplies, silage wrap, landfill covers, planter boxes, fishing nets, bundling string, seed coatings, and pellet coatings for the delayed release of pesticides, herbicides, and

\footnotetext{
* Correspondence: kitamoto@affrc.go.jp

${ }^{1}$ National Institute for Agro-Environmental Sciences (NIAES), 3-1-3 Kannondai, Tsukuba, Ibaraki 305-8604 Japan

Full list of author information is available at the end of the article
}

fertilizers (Vert 2005,). The rate of degradation of biodegradable plastics in the natural environment is controlled not only by the chemical structure of the plastic, but also by environmental conditions such as temperature, humidity, and nutrient content, all of which influence microbial activity. The rate at which a particular biodegradable plastic will degrade in a given situation is therefore still difficult to determine, and these materials often persist much longer than desired (Sakae et al. 2009). Increased use of biodegradable plastics requires greater reliability of degradation, and one means by which that might be achieved is to better understand the enzymes that efficiently degrade biodegradable plastics and the natural distributions of the microorganisms that produce these enzymes.

The main component of biodegradable mulch films is poly-butylene succinate (PBS). Several biodegradable polymers, such as poly-butylene succinate-co-adipate (PBSA), are added to control mechanical strength (Xu and Guo 2010). Three microorganisms that produce enzymes that degrade solid PBS and PBSA film are the bacterium Acidovorax delafieldii strain BS-3, which has been isolated from soil (Uchida et al. 2000), the yeast 
Cryptococcus sp. strain S-2, which is isolated from air or soil (Masaki et al. 2005), and the fungus Aspergillus oryzae (Maeda et al. 2005), which is used to produce Japanese rice-wine. However, the efficiency of isolation of microorganisms that degrade solid forms of biodegradable plastic is low. If a reliable source of microorganisms that could degrade biodegradable plastics were found, we could aim to more efficiently recruit such microorganisms to obtain more reliable rates of biodegradable plastic degradation.

Biodegradable plastics are synthesized from the polymerization of diols and dicarboxylic acids by esterification. The aerial parts of higher plants are covered by a continuous extracellular membrane of hydrophobic polymerized lipids called the cuticle. The cuticle is composed of cutin and cuticular wax: cutin is an esterified polymeric network of oxygenated C16 and C18 $\omega$ hydroxylated fatty acids (Heredia 2003,), and cuticular wax is composed of hydrocarbons. So both cutin and biodegradable plastics are composed of esterified organic acids that are solid at room temperature. The phyllosphere of healthy plants is normally colonized by bacteria, yeasts, and fungi (Lindow and Brandl 2003). Phyllosphere yeasts are primarily basidiomycete yeasts from the genera Pseudozyma, Cryptococcus, Rhodotorula, and Sporobolomyces (Allen 2006,). Hydrolytic activity (by proteases, lipases, esterase, pectinases, cellulases, and xylanases) has been observed in yeasts isolated from the phyllosphere (Ruinen 1963,, Fonseca and Inácio 2006,, Seo et al. 2007).

We observed that the chemical structures of plant surfaces were similar to those of biodegradable plastics; this led us to speculate that the cutinases or lipases from phyllosphere microorganisms might effectively degrade biodegradable plastics. From the phyllosphere, we isolated various species of Pseudozyma yeasts that degraded PBS and PBSA films. Pseudozyma spp. strains, which were easily isolated from the leaves and husks of paddy rice (Oryza sativa) and vegetables, secreted biodegradable plastic-degrading enzymes that degraded PBS or PBSA film to a greater extent than did other microorganisms.

\section{Materials and Methods}

\section{Substrates and chemicals}

To isolate biodegradable plastic-degrading yeasts from the natural environment, we used emulsified PBSA (Bionolle EM-301, average molecular weight 12 to $15 \times 10^{4}$ ). To evaluate the yeasts' solid polymer-degradation activity we used PBSA film (Bionolle 3001G), and PBS film (Bionolle 1001G), both of which has an average molecular weight 20 to $25 \times 10^{4}$ and thickness $20 \mu \mathrm{m}$. These materials were obtained from Showa Denko K. K. (Tokyo, Japan). The biodegradable plastic-degrading activity of purified enzyme was compared with that of Lipozyme CALB-L (Novozymes A/S; Krogshoejvej, Denmark), a lipase B from Candida antarctica produced by genetically modified Aspergillus niger.

\section{Microorganisms, plants, and media}

The microorganisms and rice leaves and seeds used are listed in Tables 1 and 2 . Yeast stock cultures were obtained from stock frozen at $-80^{\circ} \mathrm{C}$ and were incubated at $30^{\circ} \mathrm{C}$ for 3 days on malt-yeast-glucose-peptone (YM) agar medium containing $1 \%$ glucose, $0.5 \%$ peptone, $0.3 \%$ yeast extract, $0.3 \%$ malt extract and $1.5 \%$ agar. Seed cultures were prepared by inoculating cells grown on YM agar plates into flasks containing fungal minimum medium (FMM) with $4 \%$ glucose and incubating them at $30^{\circ}$ $\mathrm{C}$ on a rotary shaker at $220 \mathrm{rpm}$ for 4 days; the FMM was composed of $0.2 \% \mathrm{NaNO}_{3}, 0.02 \% \mathrm{MgSO}_{4}, 0.02 \%$ $\mathrm{KH}_{2} \mathrm{SO}_{4}$, and $0.1 \%$ yeast extract, dissolved in tap water before being autoclaved. For solid cultures, the seed culture $(200 \mu \mathrm{l})$ was spread on the surfaces of FMM agar plates composed of a bottom layer of FMM-agar medium $(15 \mathrm{ml})$ with no carbon source and an upper layer of $1.5 \%$ agar $(5 \mathrm{ml})$ containing a carbon source comprising $1 \%$ PBSA emulsion and $1 \%$ soybean oil (Wako Chemicals, Osaka, Japan). For liquid cultures, 500-ml Erlenmeyer flasks containing $50 \mathrm{ml}$ of FMM culture medium with one of the following carbon sources: $1 \%$ soybean oil, $6 \%$ glycerol, or $4 \%$ glucose, were inoculated with $500 \mu \mathrm{l}$ of seed culture and incubated under the same culture conditions as for seed cultures. After cultivation, dry cell weight was determined by collecting the cells from $5 \mathrm{ml}$ of culture broth, washing the pellet with the same amount of deionized water and subsequent drying at $105^{\circ} \mathrm{C}, 17 \mathrm{~h}$.

\section{Degradation of plastic films in soil}

Soil was obtained from the wheat-cropping fields of NIAES at Tsukuba, Japan. The collected soil was passed through a 2-mm sieve without prior drying. Fresh samples of the sieved soil were used for the analysis after being brought to a moisture content of $50 \%$ or $60 \%$ of saturated water capacity. Pieces of PBSA film $(2 \times 2 \mathrm{~cm})$ were packed into sterilized plastic Petri dishes $(\varphi 90 \times$ D15 mm) with $45 \mathrm{~g}$ of the moistened soil (the pieces were sandwiched between a 25-g lower layer and 20-g upper layer) and incubated at $25^{\circ} \mathrm{C}$ at constant moisture content. Three dishes with seven pieces of film in each were prepared. One piece of film was collected from each dish at intervals of 1 week for 6 weeks, and the degradation ratio of three films, collected each time, was measured by intensity modulation of luminance as follows: An image of the film was scanned with a film scanner and saved in TIFF format. The luminance of the $4-\mathrm{cm}^{2}$ area of residual film was compared with that 
Table 1 Biodegradable plastic-degrading yeasts on rice husks harvested from various areas

\begin{tabular}{|c|c|c|c|c|c|c|c|}
\hline \multirow[t]{2}{*}{$\begin{array}{l}\text { JP } \\
\text { no. }\end{array}$} & \multirow[t]{2}{*}{$\begin{array}{l}\text { Cultivar } \\
\text { name }\end{array}$} & \multirow[t]{2}{*}{ Origin } & \multicolumn{2}{|c|}{$\begin{array}{l}\text { Yeast population } \\
\text { (cfu/g) }\end{array}$} & \multirow[t]{2}{*}{$\begin{array}{l}\text { Strain } \\
\text { no }\end{array}$} & \multirow[t]{2}{*}{ Phenotype } & \multirow[t]{2}{*}{$\begin{array}{l}\text { Species } \\
\text { designation }\end{array}$} \\
\hline & & & $\overline{\mathrm{YM}}$ & PBSA & & & \\
\hline 6775 & Shinei & Hokkaido & $1 \times 10^{6}$ & $4 \times 10^{3}$ & GB-1 & Smooth & C. flavus \\
\hline 6918 & Akamai & Tohoku & $6 \times 10^{5}$ & $1 \times 10^{4}$ & GB-2 & Wrinkled & P. antarctica \\
\hline 6497 & Akinishiki & Niigata & $2 \times 10^{5}$ & $5 \times 10^{4}$ & GB-3 & Wrinkled & P. antarctica \\
\hline $203119^{1}$ & Tankei & Ibaraki & $3 \times 10^{6}$ & $5 \times 10^{5}$ & GB-4(0) & Wrinkled & P. antarctica \\
\hline \multirow[t]{2}{*}{$203119^{2}$} & Tankei & Ibaraki & $6 \times 10^{6}$ & $4 \times 10^{6}$ & GB-4(1)S & Smooth & C. laurentii \\
\hline & & & & $4 \times 10^{5}$ & GB-4(1)W & Wrinkled & P. antarctica \\
\hline \multirow[t]{2}{*}{10995} & Nourin18 & Kumamoto & $8 \times 10^{5}$ & $6 \times 10^{5}$ & GB-5S & Smooth & C. rajasthanensis \\
\hline & & & & $3 \times 10^{5}$ & GB-5W & Wrinkled & P. antarctica \\
\hline 7239 & Kanan 2 & Taiwan & $4 \times 10^{3}$ & $4 \times 10^{3}$ & GB-6 & Smooth & C. laurentii \\
\hline 5310 & Matsumae & Hokkaido & $8 \times 10^{4}$ & $8 \times 10^{4}$ & GB-7 & Wrinkled & P. antarctica \\
\hline 7032 & Ouu 52 & Akita & $8 \times 10^{5}$ & $8 \times 10^{5}$ & GB-8 & Wrinkled & P. antarctica \\
\hline 7412 & Chibanishiki & Chiba & $3 \times 10^{5}$ & $0 \times 10^{0}$ & not found & & \\
\hline \multirow[t]{2}{*}{11295} & Saikai 124 & Fukuoka & $1 \times 10^{6}$ & $4 \times 10^{4}$ & GB-10S & Smooth & C. rajasthanensis \\
\hline & & & & $4 \times 10^{3}$ & GB-10W & Wrinkled & P. antarctica \\
\hline \multirow[t]{2}{*}{8955} & Kantou 135 & Saitama & $2 \times 10^{6}$ & $1 \times 10^{6}$ & GB-11S & Smooth & C. laurentii \\
\hline & & & & $4 \times 10^{4}$ & GB-11W & Wrinkled & P. antarctica \\
\hline
\end{tabular}

All seeds were obtained from National Institute of Agrobiological Sciences (NIAS) Genebank at Tsukua, Japan

Seeds of JP nos. $203119^{1}, 5310,7032,7412,11295$, and 8955 were harvested in 2000. Those of JP nos. 6775, 6918, 6497, 203119², 10995, and 7239 were harvested in 2005.

of fresh film by using the image-processing software Aquacosmos 2.0 (Hamamatsu Photonics, Shizuoka, Japan). The degradation ratio (\%) was then calculated as:

Degradation ratio $(\%)=\frac{(\text { luminance of residual film })-(\text { luminance of fresh film })}{(\text { luminance of background })-(\text { luminance of fresh film })} \times 100$

\section{Isolation from the phyllosphere of microorganisms that} degrade biodegradable plastic emulsion

A sample of about $10 \mathrm{mg}$ of leaves or $40 \mathrm{mg}$ of rice seed husks was beaten in 20 times its weight of $10 \mathrm{mmol}$ sodium phosphate buffer ( $\mathrm{pH}$ 7.0) with a metallic cone (MC-0212; Yasui Kikai Co., Osaka, Japan) using a multibeads shocker (model MB501, Yasui Kikai Co.), with the cooling unit at $4^{\circ} \mathrm{C}$ and shaken at $1500 \mathrm{rpm}$, in 2 or 3 cycles of $30 \mathrm{~s}$ on, $30 \mathrm{~s}$ off. The suspension was diluted with the same buffer and spread on an FMM agar plate

Table 2 Isolated and stocked Pseudozyma spp. strains used in this study

\begin{tabular}{lll}
\hline Species & $\begin{array}{l}\text { Strain } \\
\text { number }\end{array}$ & Source \\
\hline P. antarctica & $\mathrm{JCM} 10317^{\top}$ & Lake sediment \\
P. rugulosa & $\mathrm{SS} 1$ & Pak choi (Brassica rapa L. var. chinensis) \\
P. aphidis & $\mathrm{SS} 2$ & Pak choi (Brassica rapa L. var. chinensis) \\
P. aphidis & $\mathrm{SS} 6$ & Mizuna (Brassica rapa L.var. nipposinica) \\
P. & $\mathrm{SS} 15$ & Ooba (Perilla frutescens Britton var. crispa \\
tsukubaensis & & Decne) \\
\hline
\end{tabular}

JCM: Japan Collection of Microorganisms of the Riken Bio-resource Center, Wako, Japan

$\mathrm{T}^{\mathrm{T}}$ : Type strain containing PBSA emulsion and soybean oil with $40 \mu \mathrm{g}$ $\mathrm{ml}^{-1}$ of chloramphenicol. The plates were incubated at $30^{\circ} \mathrm{C}$. A single colony appearing at the center of the clarified PBSA on the plate within 1 week was selected as the strain with the ability to degrade PBSA emulsion.

\section{Evaluation of degradation activity of yeast strains that degrade biodegradable plastic film}

Seed culture $(500 \mu \mathrm{l})$ of yeast strains selected for their ability to degrade PBSA emulsion on agar plates was spread onto $9 \mathrm{~cm}$-diameter FMM agar plates containing $1 \%$ PBSA emulsion and $1 \%$ soybean oil in the top layer. After incubation of the inoculated plates at $30^{\circ} \mathrm{C}$ overnight, squares of the target biodegradable plastic film (2 $\times 2 \mathrm{~cm}$ ) were mounted on the surface of the yeast lawn of the plate. After incubation of the plate at $30^{\circ} \mathrm{C}$, the films were collected at designated time intervals. The degradation ratio of films was measured as above.

\section{Identification of microorganisms that degrade biodegradable plastic}

The microorganisms isolated from phyllosphere materials were identified by rDNA sequence homology by BLAST search in the DNA Data Bank of Japan (DDBJ). rDNA sequences were obtained as follows. To extract the genomic DNA of the isolated microorganisms, cells were suspended in $100 \mu \mathrm{l}$ each of Tris-EDTA (TE)-saturated phenol and TE buffer, and were disrupted by beating with zirconia beads (Wen et al. 2005). The TE layer was used as a DNA template to amplify the rDNA 
sequence with NL1 (5'-gcatatcaataagcggaggaaaag-3') and NL4 (5'-ggtccgtgtttcaagacgg-3') as primers. The amplified DNA fragments were purified with a GeneElute PCR Clean-Up Kit (Sigma-Aldrich, St Louis, MO) and sequenced with the same primers. All DNA sequences were determined by means of a 3100 Genetic Analyzer (Applied Biosystems, Foster City, CA) using a BigDye Terminator v.3.1 cycle sequencing kit (Applied Biosystems). The nucleotide sequence of the rDNA was compared with those in the DDBJ by using the Blast search with nucleotide sequence database.

\section{Assay for activity of enzymes that degrade emulsified biodegradable plastic}

The degradation activity of enzymes on biodegradable plastic was measured in a glass test tube $(10 \mathrm{~mm}$ internal diameter) by spectrophotometry. PBSA emulsion was suspended in $2 \mathrm{ml}$ of $10 \mathrm{mmol}$ Tris- $\mathrm{HCl}$ buffer ( $\mathrm{pH}$ 6.8) containing either supernatant from the culture medium or purified enzyme solution. The percent transmittance of biodegradable plastic emulsion at a wavelength of $660 \mathrm{~nm}$ was measured as the reduction in absorbance. One unit of PBSA-degradation activity was defined as a 1-U decrease in absorbance at $660 \mathrm{~nm}$ min1. Assays for the esterase activity was performed in 96well microplates, $70 \mu \mathrm{l}$ of $50 \mathrm{mM}$ Tris- $\mathrm{HCl}(\mathrm{pH} 8.0)$ and $20 \mu \mathrm{l}$ of $1 \mathrm{mM}$ of para-nitrophenyl ( $p \mathrm{NP}$ )-butyrate or $p$ NP-palmitate (Sigma-Aldrich) in DMSO were mixed in a well. Reactions were initiated by the addition of $10 \mu \mathrm{l}$ of $\mathrm{PaE}$ (final concentration of $34 \mathrm{nM}$ ), and the reaction was carried out at $30^{\circ} \mathrm{C}$ for $5 \mathrm{~min}$, then the absorbance at $400 \mathrm{~nm}$ was measured by multi-spectrophotometer (Dainippon, Osaka, Japan). The absorbance of each substrate in the buffer without enzyme was subtracted as a blank to take into account the substrate's autohydrolysis in the solution. One unit of esterase activity was defined as the release of one micromole of $p \mathrm{NP}$ per minute.

\section{Purification of enzymes that degrade biodegradable plastic}

Liquid culture medium (500 ml of FMM with glycerol) of P. antarctica JCM 10317 in which emulsion-degrading activity had been confirmed was centrifuged at 7000 $\times g$ for $15 \mathrm{~min}$ and the supernatant was passed through a paper filter (Advantec No. 2, Toyo Roshi Kaisha, Tokyo, Japan). Ammonium sulfate powder was stirred into the filtrate to $50 \%$ saturation at $4{ }^{\circ} \mathrm{C}$, and the mixture was centrifuged at $20000 \times g$ for $15 \mathrm{~min}$. The precipitate was suspended in $20 \mathrm{mmol}$ Tris- $\mathrm{HCl}$ buffer ( $\mathrm{pH}$ 6.8) and dialyzed against the same buffer. Ion exchange chromatography of the enzyme was performed in accordance with a method for the purification of lipase and cutinase (Kolattukudy et al. 1981,, Kamini et al. 2000,, Kakugawa et al. 2001,, Akutsu-Shigeno et al. 2003) as follows. The crude enzyme was applied to a DEAESepharose Fast Flow column (GE Healthcare BioSciences, Buckinghamshire, England). The non-absorbed fraction recovered after passing through the column was applied to an SP-Sepharose Fast Flow column (GE Healthcare Bio-Sciences). After the column had been washed with the same buffer, the protein was eluted by addition of the same buffer containing $0.05 \mathrm{~mol} \mathrm{l}^{-1}$ $\mathrm{NaCl}$. The enzyme solution was concentrated by ultrafiltration (Centricut 10,000, Kurabo, Osaka, Japan) and applied to a gel-filtration column (TSK-gel G3000SW $\mathrm{XL}_{\mathrm{L}}$, Tosoh, Tokyo, Japan) with a running buffer of $50 \mathrm{mmol}$ Tris- $\mathrm{HCl}(\mathrm{pH} 6.8)$ containing $0.3 \mathrm{~mol} \mathrm{l}^{-1} \mathrm{NaCl}$. The enzymatically active fractions were collected. The protein concentration was determined with a protein assay kit (Bio-Rad Laboratories, Hercules, CA) according to the manufacturer's instructions, by using bovine serum albumin (Sigma-Aldrich) as the standard. We named the enzyme that degrades biodegradable plastic from $P$. antarctica, as PaE.

\section{Protein analysis of biodegradable plastic-degrading enzyme}

During the protein purification steps of the column chromatography, the solutions containing enzymatically active protein were separated by sodium dodecyl sulphate - polyacrylamide gel electrophoresis (SDS-PAGE) according to the method of Laemmli (1970),, using a $14.1 \%$ polyacrylamide slab gel. The gel was stained with Coomassie blue (CBB; PhastGel Blue R, GE Healthcare, Little Chalfont, England). Protein purity was confirmed by silver staining (Daiichi Kagaku Yakuhin, Tokyo, Japan) of the SDSPAGE, using a $12 \%$ gel. A single protein spot, stained with $\mathrm{CBB}$, was subjected to mass spectrometry by the method described by Shevchenko et al. (1996). Rabbit PaE polyclonal antibody was prepared from the single protein spot. The presence of PaE homolog in the culture broths from various Pseudozyma spp. was detected by using Western blot analysis with anti-PaE. Trichloroacetate (TCA)-precipitated proteins from $100 \mu \mathrm{l}$ of each culture broth were separated by means of SDS-PAGE, as above. After the transfer of the proteins to a polyvinylidene difluoride membrane (Fluorotrans, Pall, Port Washington, NY), PaE was detected according to the manufacturer's instructions of ECL detection system (GE Healthcare). The membrane was blocked with 5\% skim-milk in phosphate buffered saline with Tween 20 (PBST) and incubated overnight with $\mathrm{PaE}$ antiserum $(1 / 3000)$ at $4^{\circ} \mathrm{C}$. It was then washed with PBST and incubated with horseradish peroxidase-linked goat anti-rabbit IgG (BioRad) and examined with an ECL detection system. The E-PAGE MagicMark Unstained Protein Standard (Invitrogen, Carlsbad, California) was used for the molecular weight estimation of proteins after western blotting. 


\section{Results}

Typical rates of degradation of biodegradable plastic in soil

To determine the typical rate of biodegradation of biodegradable plastic, we incubated mulch film made from PBSA $(2 \times 2 \mathrm{~cm}$ squares $)$ in soil obtained from fields. After 4 weeks of incubation at $25^{\circ} \mathrm{C}$, the film had decomposed by $28.2 \%(\mathrm{SD}=25.2)$ at a soil moisture content of $60 \%$, and $9.1 \%(\mathrm{SD}=0.65)$ at a moisture content of $50 \%$. After 5 and 6 weeks, the degradation rates at a soil moisture content of $50 \%$ are $19.8 \%(\mathrm{SD}=4.74$ ) and $48.9 \%(\mathrm{SD}=44.3)$ (Figure 1$)$.

\section{Isolation of phyllosphere yeast strains capable of degrading biodegradable plastic film}

We observed that the chemical structures of plant surfaces are similar to those of biodegradable plastics, which led us to determine whether the microflora of plant surfaces might produce enzymes with activity against biodegradable plastics. Several strains of phyllosphere yeasts are reported to produce lipases (Ruinen 1963,, Fonseca and Inácio 2006,, Seo et al. 2007). Therefore, we speculated that the lipases from phyllosphere microorganisms might effectively degrade biodegradable plastics. For isolation of such yeasts, we used FMM agar plates containing oil and an emulsified biodegradable plastic (i.e. PBSA) in the upper layer, along with nutrients suited to the isolation of yeast. Yeast colonies that could assimilate PBSA emulsion or oil by producing lipase or esterase were expected to grow on this medium and to become surrounded by a clear zone if the

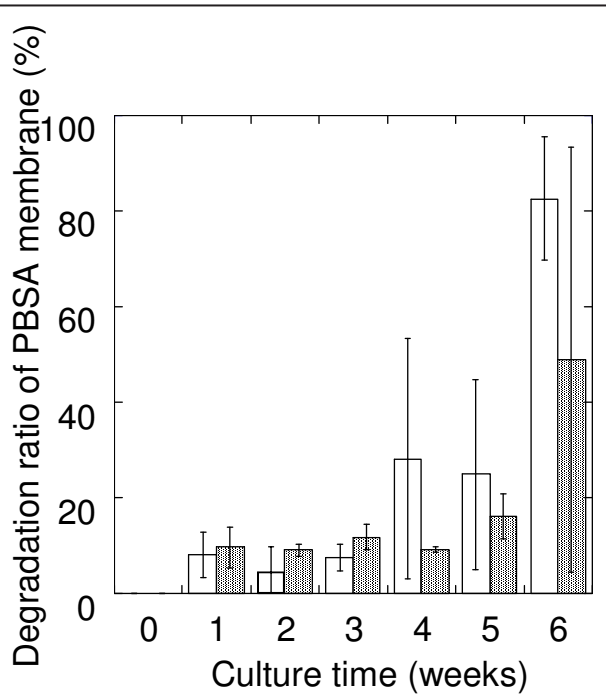

Figure 1 Degradation of PBSA mulch film in soil. Degradation of $2 \times 2 \mathrm{~cm}$ squares of PBSA mulch film packed in soil at water contents of $50 \%$ (hatched bar) and 60\% (open bar) and incubated at $25^{\circ} \mathrm{C}$. enzyme degraded the PBSA emulsion (Figure 2A). Such yeasts were isolated from two leaves of paddy rice (Figure 2A, B). The colony surfaces of yeast on each selection agar plate are uniform. Both strains were identified as $P$. antarctica, and they were named NRL-A and NRL-B. At the second screening, squares of the target biodegradable plastic film $(2 \times 2 \mathrm{~cm})$ were mounted on the surface of the yeast lawn of the plate, and observed the degradation rate as described in material and methods. Both strains degraded PBS and PBSA films on the same agar plate. As previously reported (Uchida et al. 2000,, Maeda et al. 2005,, Masaki et al. 2005), PBSA film was easier to biodegrade than PBS film.

\section{Populations and characterization of yeasts that were} found on rice husks and degraded biodegradable plastic We next examined the distribution of biodegradable plastic-degrading yeasts in the phyllosphere. We analyzed the populations of these yeast strains on the 12 stocked seed husks of 11 rice cultivars collected from various locations in Japan and Taiwan (Table 1). The yeast populations on the rice husks ranged from $4 \times 10^{3}$ to $6 \times 10^{6} \mathrm{cfu} / \mathrm{g}$. The populations of PBSA emulsiondegradable yeasts ranged from $4 \times 10^{3}$ to $4 \times 10^{6} \mathrm{cfu} / \mathrm{g}$ in 10 seed-husk collections. PBSA emulsion-degrading clones therefore constituted $2 \%$ to $100 \%$ of the yeast populations. All PBSA emulsion-degrading colonies observed in every culture have the same morphological characteristics (wrinkled or smooth), but those in Tankei 9001 (harvested in 2005), Nourin 18, Saikai 124, and Kantou 135 showed two different colony surface phenotype (wrinkled or smooth). We were unable to identify any yeast colonies in the culture from Chibanishiki because of heavy mold contamination. For detailed analysis, we picked one PBSA emulsion-degrading colony of uniform surface phenotype from each culture. All clones isolated from the wrinkled-surface colonies were identified as $P$. antarctica, and all of those from the smoothsurface colonies were identified as Cryptococcus spp. We tested the PBS and PBSA film-biodegradation activity of the isolated strains on FMM agar plates containing oil and PBSA emulsion (Figure 3A). Strains of P. antarctica are isolated from 9 of 12 stocked rice husks. And all strains of $P$. antarctica quickly degraded both PBSA and PBS films on the agar plates. Strains of $C$. laurentii also degraded PBSA film well but were slow to degrade PBS film. A strain of C. flavus slowly degraded PBSA and PBS films, but two strains of $C$. rajasthanensis did not degrade either type of film. Thus, of the 17 strains that were isolated from rice leaves or husks and degraded PBSA emulsion, 15 also degraded solid film. The type strain P. antarctica JCM 10317 also degraded both PBSA and PBS films (Figure 3A, B). Therefore, strains of $P$. antarctica are common colonizers of the surface of 


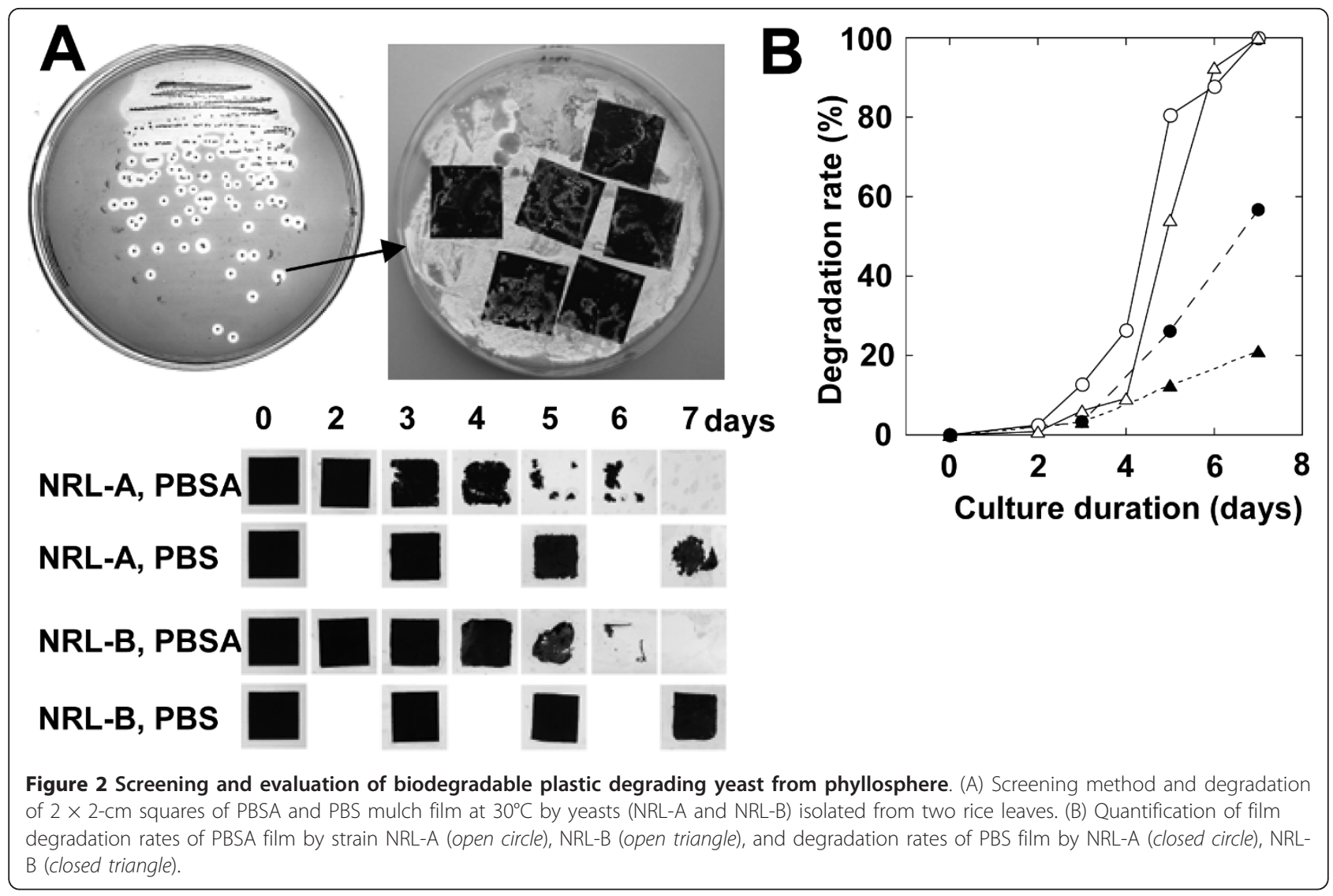

rice leaves and husks and can degrade biodegradable plastic.

\section{Biodegradable plastic-degrading activity of various species of Pseudozyma}

We speculated that species of the genus Pseudozyma other than $P$. antarctica would be able to degrade biodegradable plastics. We tested the film degradation activities of Pseudozyma spp. strains isolated from the leaves of vegetables; these were classified as $P$. rugulosa SS1, $P$. aphidis SS2, SS6, and P. tsukubaensis SS15 and were stocked at National Institute of Advanced Industrial Science and Technology (AIST) (Table 2). All of the tested strains degraded both PBSA and PBS films (Figure 3B).

\section{Culture conditions for inducing degradation of biodegradable plastics}

The next step was to analyze the culture conditions under which the isolated strains produced enzymes that could degrade biodegradable plastics. We analyzed the degradation of emulsified PBSA in FMM liquid culture medium to which soybean oil or glycerol had been added as a carbon source (Figure 4). Strains of P. antarctica, C. laurentii, and C. flavus degraded the PBSA emulsion in the presence of either carbon source. We did not detect PBSA emulsion-degrading activity in any of the culture broths of C. rajasthanensis strains. PBSA emulsion degradation activities were not detected on the vegetable strains of $P$. rugulosa, $P$. aphidis and $P$. tsukubaensis in FMM medium with oil, but weak activities were observed in FMM medium with glycerol.

Because $P$. antarctica JCM10317 strongly degraded both PBS and PBSA films and PBSA emulsion, we further analyzed the conditions under which degradation by this strain was induced. When the medium contained glucose, no degradation of PBSA was detected until after 3 days of incubation, by which time all the glucose had been consumed; PBSA degradation activity was then initiated (data not shown). The dry cell weight of the culture broth after 3 days of incubation in FMM with glucose, oil, or glycerol is $13.3,8.4$, and $7.7 \mathrm{mg} / \mathrm{ml}$, respectively. So, the enzyme activity is not dependent on growth rate. These results suggested that production of the enzymes involved in degrading biodegradable plastic was suppressed by the presence of glucose as a carbon source in the medium.

Purification and characterization of enzymes that degrade biodegradable plastics

The strain $P$. antarctica JCM10317 is synonymous with Candida antarctica CBS 5955, and known to produce 


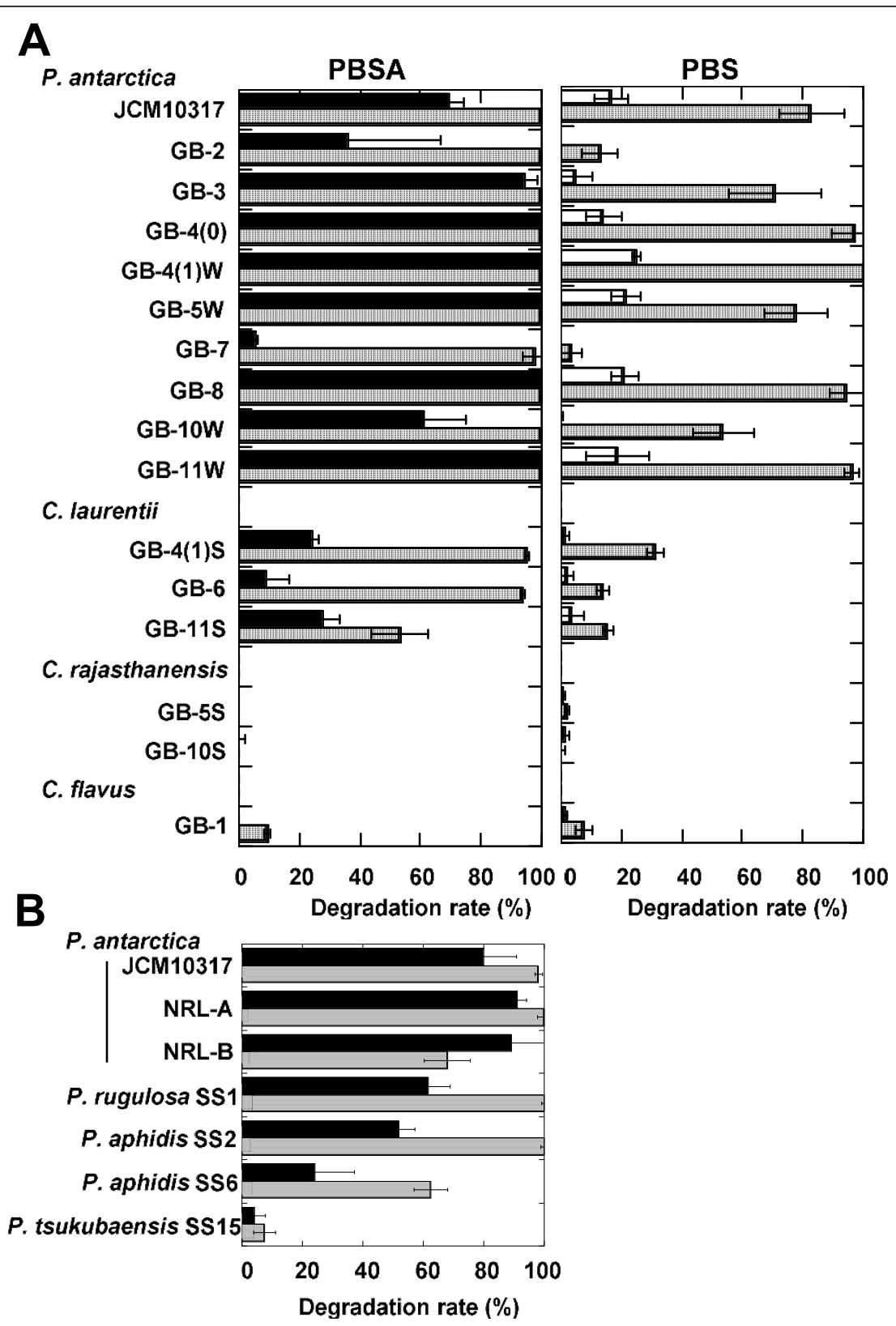

Figure 3 PBSA and PBS film-degradation activity of various species of phyllosphere yeasts. (A) PBSA and PBS film-degradation activity of yeast strains isolated from the husks of rice cultivated in various areas. Degradation rate of films after cultivation for 2 days (closed bar), 3 days (open bar), and 5 days (hatched bar), and (B) PBSA (closed bar, at 2 days) and PBS film (open bar, at 5 days)-degradation activity of various species of Pseudozyma collected from the leaves of rice and vegetables. Values are expressed as the mean (SD) at $n=3$.

two lipases, lipase A (43 kDa) and lipase B (33 kDa), on culture media containing oil (Ishii 1988). As the culture conditions required for producing lipases and $\mathrm{PaE}$ were identical, we postulated that these common lipases from the strain could degrade biodegradable plastic. However, from a culture medium of $P$. antarctica JCM10317, we purified an enzyme that degraded biodegradable plastic $(\mathrm{PaE})$ and had a molecular weight of about $22 \mathrm{kDa}$ (Table 3, Figure 5). At pH 6.8, the relative degradation activity of commercially available lipase B (CALB-L) on PBSA emulsion was about $1 / 6500$ th that of PaE. We confirmed by MS fingerprinting of $\mathrm{PaE}$ and lipase $\mathrm{B}$ that $\mathrm{PaE}$ was a different protein from lipases $\mathrm{A}$ and $\mathrm{B}$ (data not shown). Esterase activites of $\mathrm{PaE}$ against $p$ NP-butyrate and $p \mathrm{NP}$-palmitate are $715.33 \pm 124.44 \mathrm{U} / \mathrm{mg} \mathrm{PaE}$ and $194.72 \pm 36.11 \mathrm{U} / \mathrm{mg} \mathrm{PaE}$, respectively, showing that $\mathrm{PaE}$ is an esterase with wide spectrum to degrade esterified bond of long-chain fatty acids. 


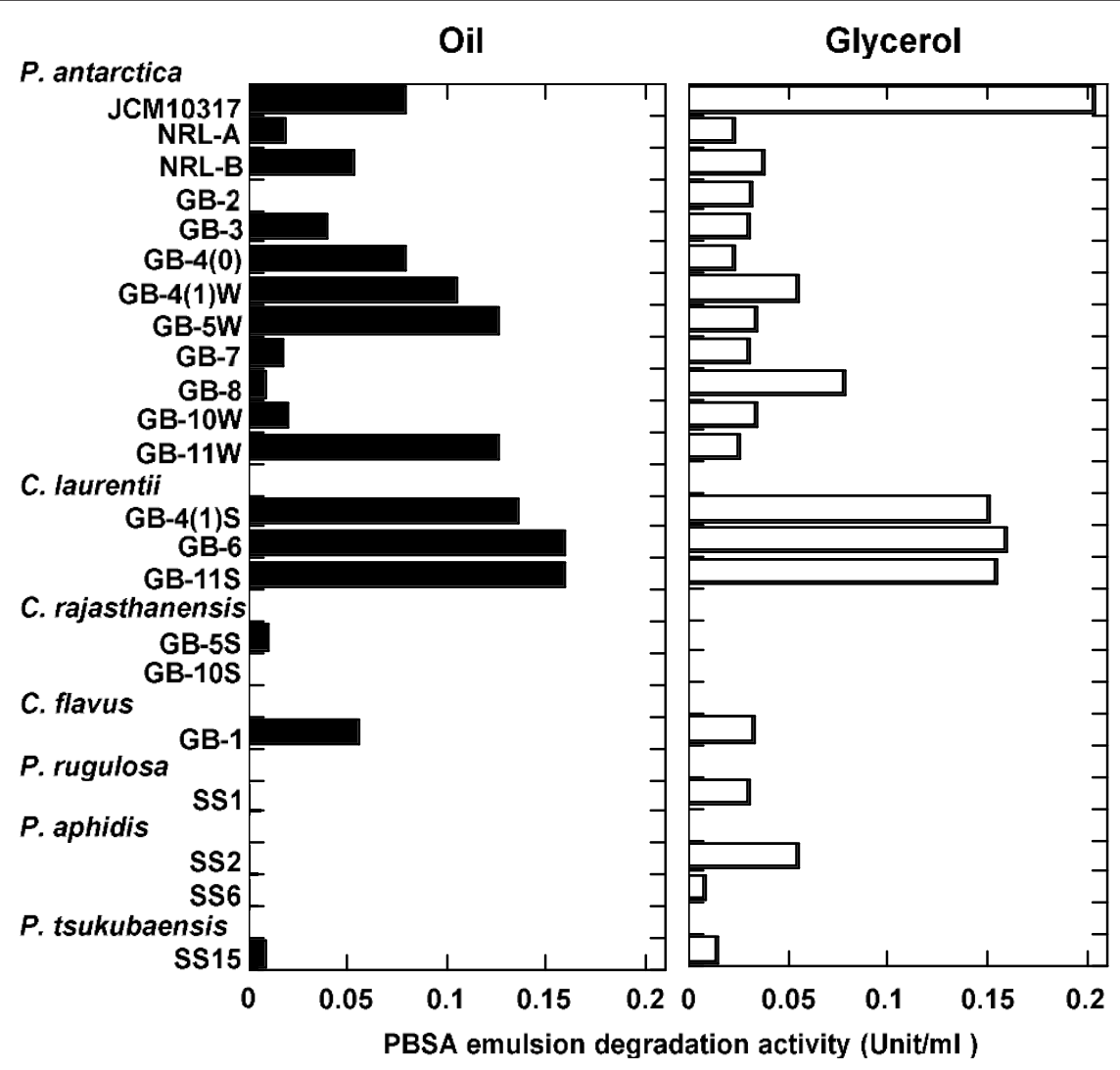

Figure 4 Degradation of PBSA emulsion by culture broths of yeast strains isolated from phyllosphere. Yeast strains were cultivated in FMM liquid medium with soybean oil or glycerol as a carbon source. After 3 days' cultivation, PBSA emulsion degradation activity was measured.

Rabbit PaE polyclonal antibody was prepared by Tanpaku Seiseikogyo (Isezaki, Japan) from the single protein spot, and confirmed the specificity of antibody to $\mathrm{PaE}$ by western blotting (Figure 6A). The immunological identity of biodegradable plastic-degrading enzymes among yeasts isolated from rice husks was analyzed by Western blotting of samples cultured for 3 days in FMM-glycerol liquid medium and incubated with antiPaE. A hybridized band with uniform mobility was detected from the culture broths of all $P$. antarctica strains, $P$. rugulosa SS1, and $P$. aphidis SS2 but no band was detected from the culture broths of $C$. laurentii (Figure 6A, B) even the PBSA emulsion degradation activities were detected (Figure 4).

\section{Discussion}

The rate of degradation of biodegradable plastics is controlled not only by their chemical structure but also by environmental conditions such as temperature, humidity and nutrient content. For example, after 4 weeks' incubation at $25^{\circ} \mathrm{C}$ in soil from a vegetable field, mulch film made from PBSA had decomposed by $28.2 \%(\mathrm{SD}=25.2$ ) at a soil moisture content of $60 \%$ but only by $9.1 \%$ (SD $=0.65$ ) at a moisture content of $50 \%$ (Figure 1). However, under the uniform environmental conditions, the degradation speed is quite variable in soil after average degradation rate over $20 \%$. For example, after 5 weeks' incubation at a soil moisture content of $50 \%$, film had decomposed by $19.8 \%(\mathrm{SD}=4.74)$ but by $48.9 \%(\mathrm{SD}=$

Table 3 Purification of biodegradable plastic-degrading enzyme from $P$. antarctica JCM10317

\begin{tabular}{lcccc}
\hline Source of fraction & $\begin{array}{c}\text { Total protein } \\
(\mathbf{m g})\end{array}$ & $\begin{array}{c}\text { Total activity } \\
(\mathbf{U})\end{array}$ & $\begin{array}{c}\text { Specific activity } \\
(\mathbf{U} / \mathbf{m g})\end{array}$ & $\begin{array}{c}\text { Yield } \\
(\%)\end{array}$ \\
\hline Culture filtrate & 23.306 & 28.96 & 1.24 & 100.0 \\
Ammonium sulfate precipitation & 0.422 & 3.42 & 8.11 & 11.83 \\
lon exchange chromatography & 0.014 & 2.73 & 198.35 & 3.44 \\
Gel filtration & 0.003 & 1.34 & 398.16 & 4.61 \\
\hline
\end{tabular}




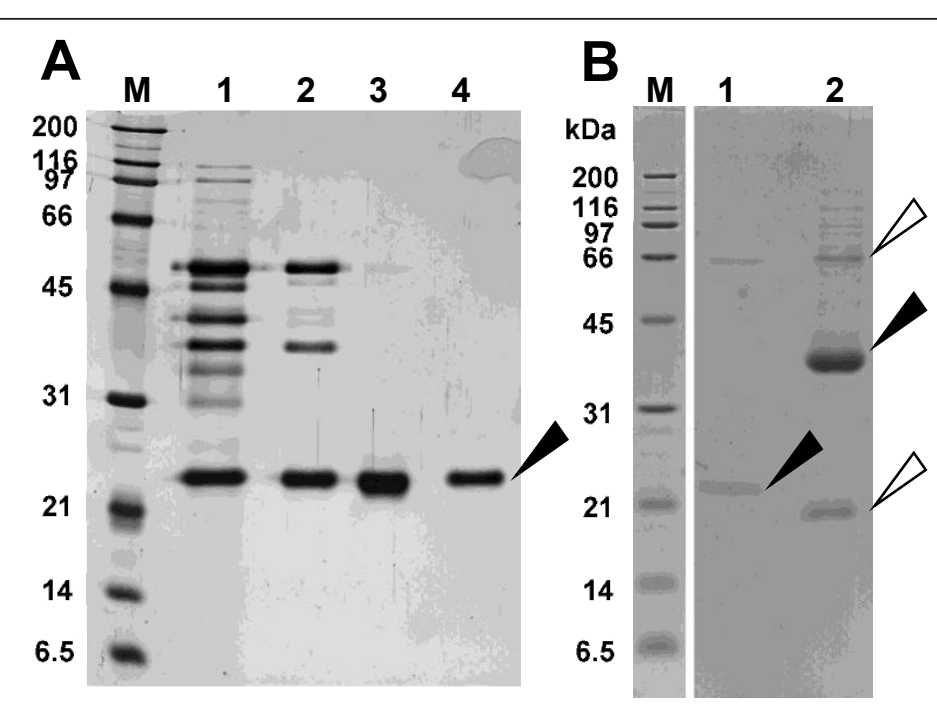

Figure 5 SDS-PAGE of biodegradable plastic-degrading enzyme (PaE) from Pseudozyma antarctica JCM 10317 and a commercially available lipase, CALB-L. (A) Purification of PaE. M Molecular mass standards; 1 Ammoniun sulfate precipitate; Fractions of $\mathbf{2}$ passed through the DEAE-Sepharose column; 3 eluated from SP-Sepharose column; 4 Gel filtration. SDS-PAGE was performed using 12\% gel and detected by

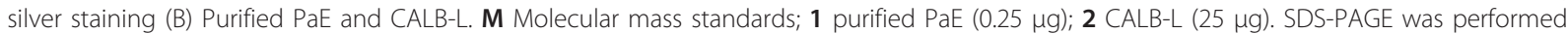
using $14.1 \% \mathrm{gel}$ and detected by CBB. The black arrow in the PaE lane shows the PaE isolated from the culture medium. The black arrow in the CALB-L lane indicates lipase B. The white arrows indicate impurities.

44.3) at 6 weeks' incubation. This shows there is considerable variability in biodegradation rates under controlled conditions by various unknown factors, and to improve the degradation reliability of biodegradable plastics we need to better understand the enzymes involved and the natural distributions of the microorganisms that produce those enzymes. However, we simply do not know where active populations of such degradative microorganisms naturally occur. Therefore, the isolation efficiency of microorganisms that degrade biodegradable plastic is low.

Microorganisms that inhabit the surfaces of natural materials can often degrade those materials in order to adhere strongly to the surface or take in the degradation products as nutrients. We observed that the structures of biodegradable plastic and cutin were similar in that

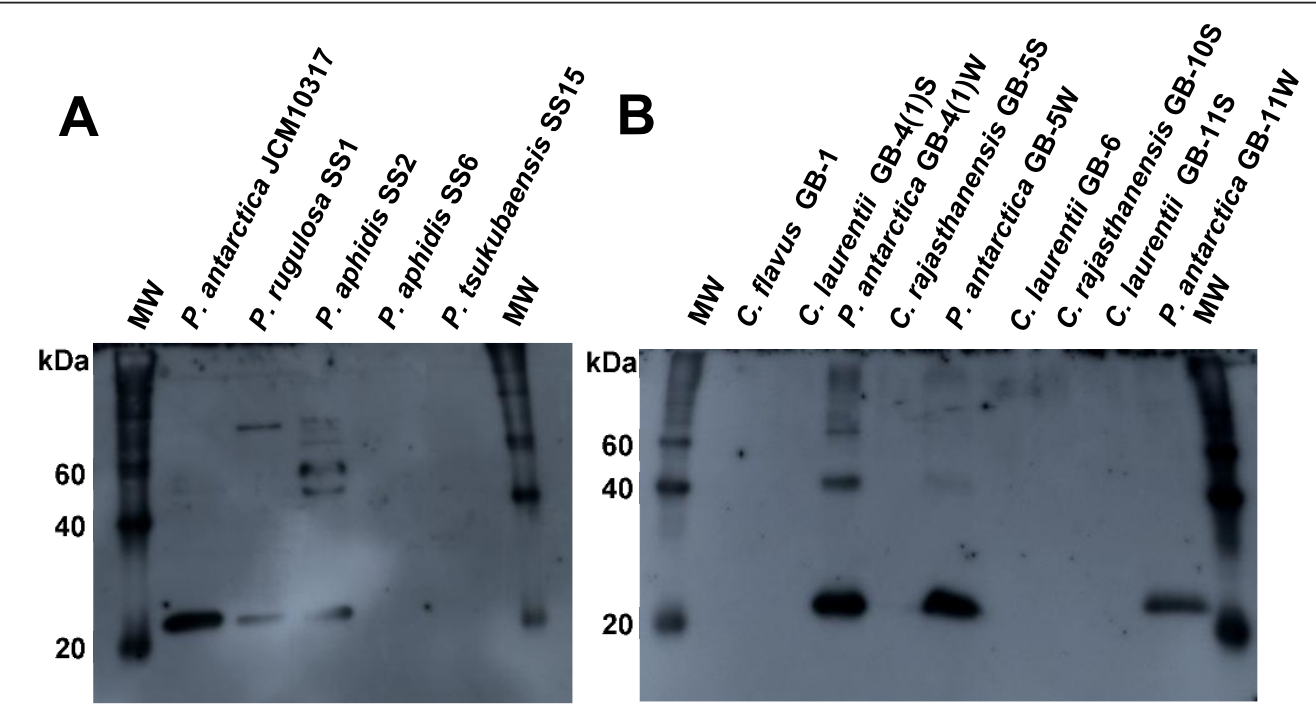

Figure 6 Immunological identification of biodegradable plastic-degrading enzymes of phyllosphere yeasts. Yeasts from vegetables (A) and rice husks (B) were cultivated in FMM liquid medium with glycerol for 3 days. TCA-precipitated culture broth (100 $\mu$ l) was separated by using SDS gel electrophoresis and analyzed by use of Western blotting with anti-PaE. M Molecular mass standards. 
both were made from esterified organic acids in solid form at room temperature. In light of the lipase and esterase activities of phyllosphere yeasts, we first attempted to isolate phyllosphere yeasts that could degrade emulsified biodegradable plastic. As strains of Fusarium sp. and Pseudozyma spp. produce lipase on media containing natural oils as the sole carbon sources (Ishii 1988,, Feng et al. 2005), we speculated that the lipase or esterase from phyllosphere microorganisms might effectively degrade biodegradable plastics.

We were successful in isolating yeasts that degrade biodegradable plastics from the phyllosphere on minimum medium agar plates containing oil and emulsified PBSA. We found that the two yeasts isolated from two leaves of paddy rice on these plates could degrade biodegradable plastic emulsion (Figure 2A, B), and from the seed husks of 11 of 12 rice cultivars collected from various locations (Table 1). The isolation efficiency is 2 to $100 \%$ of phyllosphere yeast population. Of the 17 strains that were isolated from rice leaves or husks and degraded PBSA emulsion, 15 also degraded solid film; in contrast, when we isolated biodegradable plastic-degrading bacteria from soil or sediment (Uchida et al. 2000), only $1 \%$ to $2 \%$ of the bacteria that degraded the PBSA emulsion degraded solid film (unpublished data). These results show that the populations of biodegradable plastic-degrading yeasts in the phyllosphere were quite high in the natural environment. Strains of $P$. antarctica with strong PBS and PBSA film degradation activities are isolated among 9 of 12 rice cultivars. These results showed that strains of $P$. antarctica are common colonisers of the surfaces of rice leaves and husks and are capable of degrading biodegradable plastics. Yeast strains of the genus Pseudozyma are often isolated from various plant surfaces (Allen 2006). We therefore speculated that species of the genus Pseudozyma other than P. antarctica may be able to degrade biodegradable plastics. All the tested strains of Pseudozyma spp., isolated from phyllosphere and stocked in our collection, degraded PBS and PBSA films (Figure 3B). Of the tested strains, the type strain P. antarctica JCM10317 demonstrated the strongest degradation activity on PBS and PBSA mulch film (Figure 3A, B). A strain of Candida antarctica CBS 5955 (a synonym of $P$. antarctica JCM 10317) is known to produce two lipases, lipase A (43 kDa) and lipase B (33 kDa), on culture media containing oil (Ishii 1988). Thus we speculated such lipases may degrade biodegradable plastics. However, from a culture medium of $P$. antarctica JCM10317, we purified an enzyme that degraded biodegradable plastic (PaE) and had a molecular weight of about $22 \mathrm{kDa}$ (Figure 5). The relative degradation activity of PBSA emulsion by lipase $B$ (CALB-L) was about $1 / 6500$ th of that of PaE. We have confirmed that $\mathrm{PaE}$ was a different protein from lipase
A and B. Furthermore, we have observed that all isolated Pseudozyma spp. strains, which showed biodegradable plastic film degradation ability, secreted immunologically identical biodegradable plastic-degrading enzyme; but there may be the structural differences in enzymes from Cryptococcus spp. between PaE (Figure 6 ). These results demonstrated that the strains of the genus Pseudozyma isolated in this study degrade biodegradable plastics using a novel enzyme. In order to accelerate the degradation of biodegradable plastic wastes, we therefore propose that they be treated with these phyllosphere yeasts or their enzymes. Further studies should be conducted to characterize these yeasts and their enzymes as well as to develop practical methods for utilizing them.

\section{Acknowledgements}

We thank H. lefuji, K. Gomi, and F. Takeshi for their comments on the research and Showa Denko K. K. and Novozymes for materials. This research was financially supported by the Japan Science and Technology Agency, Ministry of the Environment, KAKENHI (235658083), and the National Institute for Agro-Environmental Sciences, Japan.

\section{Author details}

${ }^{1}$ National Institute for Agro-Environmental Sciences (NIAES), 3-1-3 Kannondai, Tsukuba, Ibaraki 305-8604 Japan ${ }^{2}$ Research Institute for Innovation in Sustainable Chemistry, National Institute of Advanced Industrial Science and Technology (AIST), Tsukuba Central 5-2, Higashi 1-1-1, Tsukuba, Ibaraki 3058565, Japan ${ }^{3}$ National Institute of Agrobiological Sciences (NIAS), 2-1-2 Kannondai, Tsukuba, Ibaraki 305-8602, Japan ${ }^{4}$ Graduate School of Life and Environmental Sciences, University of Tsukuba, Tsukuba, 305-8572 Ibaraki, Japan $4{ }^{5}$ Institute of Biogeosciences, Japan Agency for Marine-Earth Science and Technology (JAMSTEC), 2-15, Natsushima-cho, Yokosuka 237-0061, Japan

\section{Competing interests}

The authors declare that they have no competing interests.

Received: 6 October 2011 Accepted: 29 November 2011 Published: 29 November 2011

\section{References}

Akutsu-Shigeno Y, Teeraphatpornchai T, Teamtisong K, Nomura N, Uchiyama H, Nakahara T, Nakajima-Kambe T (2003) Cloning and sequencing of a poly(DLlactic acid) depolymerase gene from Paenibacillus amylolyticus strain TB-13 and its functional expression in Escherichia coli. Appl Environ Microbiol 69:2498-2504. doi:10.1128/AEM.69.5.2498-2504.2003.

Allen TW, Burpee LL, Buck JW (2006) Variable adhesion and diurnal population patterns of epiphytic yeasts on creeping bentgrass. Can J Microbiol 52:404-410. doi:10.1139/w05-145.

Feng J, Liu G, Selvaraj G, Hughes GR, Wei Y (2005) A secreted lipase encoded by LIP1 is necessary for efficient use of saturated triglyceride lipids in Fusarium graminearum. Microbiology 151:3911-3921. doi:10.1099/mic.0.28261-0.

Fonseca Á, Inácio J (2006) Phylloplane Yeasts. Biodiversity and ecophysiology of yeasts. Springer, Berlin, London

Heredia A (2003) Biophysical and biochemical characteristics of cutin, a plant barrier biopolymer. Biochim Biophys Acta Gen Subj 1620:1-7. doi:10.1016/ S0304-4165(02)00510-X

Ishii M (1988) Positionally non-specific lipase from Candida sp., a method for producing it, its use and a recombinant DNA process for produce it. International applications published under the Patent Cooperation Treaty, Swedish Patent Office W088/02775

Kakugawa K, Shobayashi M, Suzuki O, Miyakawa T (2002) Purification and characterization of a lipase from the glycolipid-producing yeast Kurtzmanomyces sp. 1-11. Biosci Biotechnol Biochem 66:978-985. doi:10.1271/ bbb.66.978. 
Kamini NR, Fujii T, Kurosu T, lefuji H (2000) Production, purification and characterization of an extracellular lipase from the yeast, Cryptococcus sp. S-2. Process Biochem 36:317-324. doi:10.1016/\$0032-9592(00)00228-4.

Kolattukudy PE, Purd RE, Maiti IB (1981) Cutinases from fungi and pollen. Methods Enzymol 71:652-664

Laemmli U (1970) Cleavage of structural proteins during the assembly of the head of bacteriophage T4. Nature 277:680-685

Lindow SE, Brandl MT (2003) Microbiology of the phyllosphere. Appl Environ Microbiol 69:1875-1883. doi:10.1128/AEM.69.4.1875-1883.2003.

Maeda H, Yamagata Y, Abe K, Hasegawa F, Machida M, Ishioka R, Gomi K, Nakajima T (2005) Purification and characterization of a biodegradable plastic-degrading enzyme from Aspergillus oryzae. Appl Microbiol Biotechnol 67:778-788. doi:10.1007/s00253-004-1853-6.

Masaki K, Kamini NR, Ikeda H, lefuji H (2005) Cutinase-like enzyme from the yeast Cryptococcus sp. strain S-2 hydrolyzes polylactic acid and other biodegradable plastics. Appl Environ Microbiol 71:7548-7550. doi:10.1128/ AEM.71.11.7548-7550.2005.

Ruinen J (1963) The phyllosphere. II. Yeasts from the phyllosphere of tropical foliage. Anton Van Leeuw 29:425-38. doi:10.1007/BF02046095.

Sakae Y, Matsubara T, Aida M, Kondo H, Masaki K, lefuji H (2009) ONIOM Study of the mechanism of the enzymatic hydrolysis of biodegradable plastics. Bull Chem Soc Jpn 82:338-346. doi:10.1246/bcs.82.338.

Seo HS, Um HJ, Min J, Rhee SK, Cho TJ, Kim YH, Lee J (2007) Pseudozyma jejuensis sp. nov., a novel cutinolytic ustilaginomycetous yeast species that is able to degrade plastic waste. FEMS Yeast Res 7:1035-45. doi:10.1111/j.15671364.2007.00251.x.

Shevchenko A, Wilm M, Vorm O, Mann M (1996) Mass spectrometric sequencing of proteins silver-stained polyacrylamide gels. Anal Chem 68:850-858. doi:10.1021/ac950914h.

Uchida H, Nakajima-Kambe T, Shigeno-Akutsu Y, Nomura N, Tokiwa Y, Nakahara T (2000) Properties of a bacterium which degrades solid poly (tetramethylene succinate)-co-adipate, a biodegradable plastic. FEMS Microbiol Lett 189:25-29. doi:10.1111/j.1574-6968.2000.tb09201.x.

Vert M (2005) Aliphatic polyesters: great degradable polymers that cannot do everything. Biomacromolecules 6:538-546. doi:10.1021/bm0494702.

Wen Y, Hatabayashi H, Arai H, Kitamoto HK, Yabe K (2005) Function of the cypX and moxY genes in aflatoxin biosynthesis in Aspergillus parasiticus. Appl Environ Microbiol 71:3192-3198. doi:10.1128/AEM.71.6.3192-3198.2005.

Xu J, Guo BH (2010) Poly(butylene succinate) and its copolymers: research, development and industrialization. Biotechnol J 5:1149-63. doi:10.1002/ biot.201000136.

doi:10.1186/2191-0855-1-44

Cite this article as: Kitamoto et al:: Phyllosphere yeasts rapidly break down biodegradable plastics. AMB Express 2011 1:44.

\section{Submit your manuscript to a SpringerOpen ${ }^{\circ}$ journal and benefit from:}

- Convenient online submission

- Rigorous peer review

- Immediate publication on acceptance

- Open access: articles freely available online

- High visibility within the field

- Retaining the copyright to your article

Submit your next manuscript at $\gg$ springeropen.com 